\title{
COUNTING BERG PARTITIONS VIA STURMIAN WORDS AND SUBSTITUTION TILINGS
}

\author{
ARTUR SIEMASZKO AND MACIEJ P. WOJTKOWSKI
}

\begin{abstract}
We develop the connection of Berg partitions with special substitution tilings of two tiles. We obtain a new proof that the number of Berg partitions with a fixed connectivity matrix is equal to half of the sum of its entries, $\mathrm{S}-\mathrm{W}$.

This approach together with the formula of Séébold [Seb], for the number of substitutions preserving a given Sturmian sequence, shows that all of the combinatorial substitutions can be realized geometrically as Berg partitions.

We treat Sturmian tilings as intersection tilings of bi-partitions. Using the symmetries of bi-partitions we obtain geometrically the palindromic properties of Sturmian sequences (Theorem 3) established combinatorially by de Luca and Mignosi, [L-M].
\end{abstract}

\section{INTRODUCTION}

Let us consider the interval exchange map of two intervals, $J_{1}$ and $J_{2}$, with lengths $s_{1}$ and $s_{2}$ respectively. We denote the map by $h$ : $J^{s} \rightarrow J^{s}, J^{s}=J_{1} \cup J_{2}$. The discontinuity of the map is superficial. Indeed, when the endpoints of $J^{s}$ are identified, we obtain the rotation of the circle with the rotation number $\frac{s_{1}}{s_{1}+s_{2}}$, or $\frac{s_{2}}{s_{1}+s_{2}}$, depending on the order of the intervals. Let us further consider the suspension flow of $h$ with the roof function constant on $J_{1}$ and $J_{2}$, and equal to $u_{1}$ and $u_{2}$ respectively. We obtain the flow $H^{t}: R_{1} \cup R_{2} \rightarrow R_{1} \cup R_{2}, t \in \mathbb{R}$, where $R_{1}=\left[0, s_{1}\right] \times\left[0, u_{1}\right]$ and $R_{2}=\left[-s_{2}, 0\right] \times\left[0, u_{2}\right]$. Again the discontinuity of $H^{t}$ is superficial.

Let $L \subset \mathbb{R}^{2}$ be the lattice generated by two translations $e_{0}=\left(s_{2}, u_{1}\right)$ and $f_{0}=\left(-s_{1}, u_{2}\right)$. Translations of the rectangles $R_{1}$ and $R_{2}$ by vectors from the lattice $L$ form a 2-periodic tiling of the plane. Hence $R_{1} \cup R_{2}$ is a fundamental domain of the torus $\mathbb{T}^{2}=\mathbb{R}^{2} / L$.

Partition of the torus into $\left\{R_{1}, R_{2}\right\}$ is called a bi-partition. The union of their horizontal sides in the torus $\mathbb{T}^{2}$ is covered by the interval of the horizontal axis $J^{s}=\left[-s_{2}, s_{1}\right]$, the horizontal spine. The union of their vertical sides is covered by the interval of the vertical axis

2010 Mathematics Subject Classification. 37D.

Key words and phrases. Toral automorphisms, Markov partitions, Berg partitions, Sturmian sequences, tilings, substitutions .

Research partially supported by the NCN grant 2011/03/B/ST1/04427 . 
$J^{u}=\left[0, u_{1}+u_{2}\right]$, the vertical spine. Let us note that $J^{s} \cap J^{u}$ contains four points in the torus, two endpoints of the horizontal spine, and two endpoints of the vertical spine.

The flow $H^{t}$ is given by the constant vertical vector field in the torus $\mathbb{T}^{2}$. Indeed for this field the return map to the horizontal spine $J^{s}=J_{1} \cup J_{2}$ is equal to the interval exchange transformation.

Further let $(x, y)$ be the Cartesian coordinates in $\mathbb{R}^{2}$ and $(\xi, \eta)$ be the coordinates associated with the basis $\left(e_{0}, f_{0}\right)$, i.e., $x=s_{2} \xi-s_{1} \eta, y=$ $u_{1} \xi+u_{2} \eta$. We get $\dot{\xi}=s_{1} d^{-1}, \dot{\eta}=s_{2} d^{-1}$, where $d=s_{1} u_{1}+s_{2} u_{2}$. In particular we observe that the slope of the trajectory in the $(\xi, \eta)$ coordinates is equal to $s_{2} s_{1}^{-1}$ and hence it is independent of the roof function.

For a given hyperbolic matrix $F \in G L(2, \mathbb{Z})$ with non-negative entries, let $\left(s_{1}, s_{2}\right)$ and $\left(u_{1}, u_{2}\right)$ be, respectively, the column and the row eigenvectors with positive entries. The automorphism $\mathcal{F}$ of the torus $\mathbb{T}^{2}$ defined in the basis $\left(e_{0}, f_{0}\right)$ by $F$ preserves the horizontal and vertical lines, i.e., it is given by a diagonal matrix in the Cartesian coordinates $(x, y)$, $\mathrm{S}-\mathrm{W}]$. It follows that the bi-partition $\left\{R_{1}, R_{2}\right\}$ is a Berg partition, i.e. it has the Markov property. Indeed in the case of $F$ with both positive eigenvalues $\left(\operatorname{det} F=1\right.$ ) we get $\mathcal{F}\left(J^{u}\right) \supset J^{u}$ and $\mathcal{F}\left(J^{s}\right) \subset J^{s}$. These inclusions hold because both spines contain the origin, which is a fixed point of $\mathcal{F}$.

It can be checked that the last inclusion holds also in the case of negative stable eigenvalue, and we will prove it later on.

Hyperbolic automorphisms have in general many fixed points. Translating the bi-partition $\left\{R_{1}, R_{2}\right\}$ so that both spines contain a fixed point of $\mathcal{F}$, we obtain nonequivalent Berg partitions with the same connectivity matrix $F^{T}$, at least in the case of both positive eigenvalues. It was proven in $[\mathrm{S}-\mathrm{W}]$ that

Theorem 1. The number of nonequivalent Berg partitions for $\mathcal{F}$ with the connectivity matrix $F^{T}$ is equal to $\left[\frac{\sigma}{2}\right]$, where $\sigma$ is the sum of all entries of $F$, and [.] denotes the integer part of a number.

The formula holds also for the toral automorphisms $\mathcal{F}$ defined by $-F$ in the basis $\left(e_{0}, f_{0}\right)$. The proof given in $[\mathrm{S}-\mathrm{W}]$ has three distinct cases of eigenvalue signs, with different geometry, and the common value for the number of Berg partitions was somewhat surprising. In this paper we give another proof of Theorem 1, based on the connection of Berg partitions with 1-dimensional substitution tilings, $[\mathrm{PF}]$.

The 1-dimensional tilings defined by the intersection of vertical lines with a bi-partition are associated with infinite Sturmian sequences. If the bi-partition is a Berg partition then the tilings are preserved under an appropriate substitution.

The number of different substitutions preserving a given Sturmian sequence was established by Séébold [Seb] to be equal to the sum of 
entries of $F$ minus one. It is essentially the same number as the number of nonequivalent Berg partitions from Theorem 1. The difference arises from the fact that reversed substitutions come from equivalent Berg partitions.

This equality shows that all combinatorial substitutions of Sturmian words can be realized geometrically as toral automorphisms acting on respective Berg partitions. It allows the translation of combinatorial questions regarding substitutions of Sturmian words into the geometric language. We describe this connection in Section 5. Let us note that although we have two essentially identical formulas they do not follow formally from each other. A priori one can only claim that the number of Berg partitions does not exceed the number of substitutions.

In Sections 2 and 3 we develop the geometric approach to Sturmian sequences as intersection tilings of 2-periodic tilings of the plane. Using the symmetries of the 2-periodic tilings we give a geometric proof of the palindromic properties of Sturmian sequences which were established combinatorially by de Luca and Mignosi, [L-M]. These properties are crucial in the counting of Berg partitions obtained in Section 4.

\section{INTERSECTION TILINGS}

Given a bi-partition $\left\{R_{1}, R_{2}\right\}$, as described above, we get the 2periodic tiling of $\mathbb{R}^{2}$ by the rectangles $R_{1}+L$ and $R_{2}+L$. We will consider the intersection tilings of vertical lines in $\mathbb{R}^{2}$. Namely, for a given vertical line $l$, intersecting the horizontal spine $J^{s}$, we consider its partition by the intersection with the rectangles of the 2-periodic tiling. Another way to describe the intersection tiling is to cut the line $l$ by the translates of the horizontal spine $J^{s}+L$. The lengths of these intervals (tiles) are $u_{1}$ and $u_{2}$, and we will assume for convenience that $u_{1} \neq u_{2}$.

We describe such a tiling by a sequence $\omega \in \Omega=\{a, b\}^{\mathbb{Z}}$, where the letters $a$ and $b$ correspond to the intersection with the translates of $R_{1}$ and $R_{2}$, respectively. For a sequence $\omega=\left\{c_{n}\right\}$ the symbol $c_{0}$ corresponds to the tile with the lower endpoint on the horizontal spine $J^{s}$.

The set of sequences describing intersection tilings for a given bipartition will be denoted by $\mathcal{T} \subset \Omega$. The sequences in $\mathcal{T}$ are very special. Several combinatorial descriptions are available. A particularly friendly introduction into the subject can be found in the paper of Series [Ser]. We will elaborate some of these properties in what follows.

Let us note that the space of tilings $\mathcal{T} \subset \Omega$ is invariant under the shift. It can be identified with the horizontal spine $J^{s}$, and the shift becomes the interval exchange map $h: J^{s} \rightarrow J^{s}$. The vertical lines which intersect translates of the vertical spine $J^{u}+L$ give rise to two tilings differing by the exchange of $[a, b]$ and $[b, a]$. Indeed the intersection with $J^{u}+z, z \in L$ can be interpreted as the intersection with first 
$R_{1}+z$ and then $R_{2}+z$ (intersection on the "right side"), or the other way around (intersection on the "left side"). In this way two sequences from $\mathcal{T}$ are glued into one point in $J^{s}$.

The study of such 1-dimensional tilings is thus equivalent to the analysis of the symbolic dynamics for the rotation $h$ and the partition $J^{s}=J_{1} \cup J_{2}$. It goes back to the papers of Hedlund and Morse [H-M1, [H-M2]. Their Sturmian sequences coincide with our intersection tilings up to countably many nontransitive sequences.

We can further introduce the tiling space $R_{1} \cup R_{2}$, as the suspension of the shift on $\mathcal{T}$. It carries the Kronecker flow $H^{t}$. Both of these tiling spaces come with respective invariant Lebesgue measures. They have their distinct advantages and we will freely use one or the other.

If the rotation number $\frac{s_{1}}{s_{1}+s_{2}}$ is rational then there are only finitely many tilings, shifts of a single periodic tiling. Indeed in such a case the lattice $L$ contains a vertical vector, and the corresponding translation preserves the 2-periodic tiling of $\mathbb{R}^{2}$, and all the intersection tilings.

The structure of the periodic tiling is far from arbitrary. We will elaborate on its structure in the following.

In the irrational case there are continuum of different tilings. For simplicity of formulations we will assume that $\frac{s_{2}}{s_{1}}$ is irrational, and we include some comments on the rational case. In the irrational case two tilings are the same up to the shift if and only if one of the lines is a translate of the other by a vector from the lattice $L$. Indeed if a translation takes one tiling into another then the closure in $\mathbb{T}^{2}$ of the set of all $a$-tiles from the first line is mapped by the translation into the closure of the set of all $a$-tiles in the second line. These closures are equal to $R_{1}$ and hence the translation vector must belong to the lattice $L$.

\section{Symmetry of the INTERSECTION TILINGS}

The group of symmetries preserving the 2-periodic tiling of the plane, by the translates of $R_{1}$ and $R_{2}$, contains translations from $L$. It also contains rotations by $\pi$ around the centers of the vertical and horizontal spines, and the centers of the rectangles $R_{1}, R_{2}$, or their translations by $L$. The set of fixed points of such rotations can be described as $\left(0, \frac{1}{2}\left(s_{1}-s_{2}\right)\right)+\frac{1}{2} L$. Indeed, the rotation by $\pi$ around the center of $J^{s}$ has four fixed points in the torus $\mathbb{T}^{2}$ : the centers of $J^{s}$ and $J^{u}$, and the centers of $R_{1}$ and $R_{2}$. Moreover for any two copies of $J^{u}$ in the plane $\mathbb{R}^{2}$ the rotation by $\pi$ which exchanges them, preserves the intersection $J^{s} \cap J^{u} \subset \mathbb{T}^{2}$, and hence also the horizontal spine $J^{s}$. It follows that this rotation takes a copy of $J^{s}$ in $\mathbb{R}^{2}$ into another copy, and thus preserves the 2-periodic tiling of $\mathbb{R}^{2}$.

The vertical line through the origin, and its translations by vectors from $L$ have two tilings, arising from two tilings of the vertical spine, 
$[a, b]$ and $[b, a]$. These tilings will be called principal, and their ambiguous part where the vertical line intersects a translation of the vertical spine will be called the lock. The principal tiling $\left\{c_{i}\right\} \in \mathcal{T}$ is unique up to the shift and the change of the lock described above. Further it is palindromic in the sense that if the lock is equal to $\left[c_{k}, c_{k+1}\right]$ then $c_{k-r}=c_{k+1+r}$ for $r=1,2, \ldots$. Indeed, for a line $l$ with the principal tiling we rotate the plane by $\pi$ around the center of the copy of the vertical spine $J^{u}$ in our line $l$. The line $l$ and the 2-periodic tiling are preserved, and the intersection tiling is reversed, except for the lock.

More generally we say that a 1-dimensional tiling $\left\{c_{i}\right\} \in \Omega$ is almost palindromic if there are integers $k, l$ such that $c_{k-r}=c_{l+r}$ for $r=1,2, \ldots$.

Proposition 2. If an intersection tiling is almost palindromic then it must be the principal tiling or, up to the shift, the tiling of one of three lines passing through the centers of $R_{1}, R_{2}$ and $J^{s}$.

Proof. Consider a line $l$ with an almost palindromic tiling and the rotation by $\pi$ around the point in $l$ which realizes the symmetry of the tiling. In particular it exchanges the respective points of intersections of $l$ with translates of $J^{s}$. This rotation factors to the torus, and the set of intersection points (the endpoints of tiles exchanged by the rotation) projects to a dense subset of $J^{s} \subset \mathbb{T}^{2}$. It follows that the rotation of $\mathbb{T}^{2}$ takes $J^{s}$ onto itself, and hence it preserves the bi-partition. Thus the line $l$ contains the translate of one of the four centers of $J^{s}, J^{u}, R_{1}, R_{2}$.

Up to the shift there are exactly five almost palindromic tilings, two containing $J^{u}$ (the lock), and three actually palindromic through the centers of $J^{s}, R_{1}$ and $R_{2}$.

While there are only five "global" palindromes among the intersection tilings, there is an abundance of "local" palindromes. Let us consider bases $\left(e_{i}, f_{i}\right), i \geq 0$ from the fan of bases, defined by the horizontal and vertical axes, and the lattice $L$. Let us recall $([S-W])$ that the fan of bases contains all the bases $(e, f)$ of $L$ such that $e$ is in the first quadrant, and $f$ is in the second quadrant. The fan of bases is described by the cutting sequence $\left(s_{i}\right), s_{i} \in\{0,1\}$, and it is exhausted by the sequence of bases $\left(e_{i}, f_{i}\right), i \in \mathbb{Z}$, where $e_{i+1}=e_{i}+f_{i}, f_{i+1}=f_{i}$ if $s_{i}=1$, and $e_{i+1}=e_{i}, f_{i+1}=e_{i}+f_{i}$ if $s_{i}=0$. We have

$\left[\begin{array}{ll}e_{i} & f_{i}\end{array}\right]=\left[\begin{array}{ll}e_{0} & f_{0}\end{array}\right]\left[\begin{array}{cc}k_{i} & l_{i} \\ m_{i} & n_{i}\end{array}\right], \quad$ where $\quad\left[\begin{array}{cc}k_{i} & l_{i} \\ m_{i} & n_{i}\end{array}\right] \in S L(2, \mathbb{Z})$,

and $k_{i}, l_{i}, m_{i}, n_{i} \geq 0$ for $i \geq 0$.

Theorem 3. For a basis $\left(e_{i}, f_{i}\right), i \geq 2$, let $p=k_{i}+m_{i}, r=l_{i}+n_{i}$, and $p \geq 2, r \geq 2$. In the principal tiling $\left\{c_{j}\right\}$ with the lock $\left[c_{0}, c_{1}\right]$ the three words $\left(c_{2}, \ldots, c_{p-1}\right),\left(c_{2}, \ldots, c_{r-1}\right),\left(c_{2}, \ldots, c_{p+r-1}\right)$ are palindromes. 
Moreover

$$
\begin{aligned}
\left(c_{2}, \ldots, c_{p+r-1}\right) & =\left(c_{2}, \ldots, c_{p-1}, b, a, c_{2}, \ldots, c_{r-1}\right) \\
& =\left(c_{2}, \ldots, c_{r-1}, a, b, c_{2}, \ldots, c_{p-1}\right) .
\end{aligned}
$$

The properties of Sturmian words from this theorem were formulated by de Luca and Mignosi in [L-M].

For any relatively prime numbers $p$ and $r$ there is exactly one palindromic word $\left(c_{2}, \ldots, c_{p+r-1}\right)$ such that $\left(c_{2}, \ldots, c_{p-1}\right)$ and $\left(c_{2}, \ldots, c_{r-1}\right)$ are also palindromic. By exactly one we mean up to the exchange of the symbols $a$ and $b$. This fact was formulated by Raphael M. Robinson $[\mathrm{R}]$ and proven, among others, by Pedersen $[\mathrm{P}]$.

Proof. Let us consider all the lattice points in the first quadrant, and its convex hull. The boundary of the convex hull (the Klein's sail) contains lattice points (i.e., points from $L$ ). They can be described using the fan of bases $\left(e_{j}, f_{j}\right), j \in \mathbb{Z}$, associated with the lattice $L$ and the horizontal and vertical axes, $[\mathrm{S}-\mathrm{W}]$. Namely the set of points on the boundary of the convex hull is equal to $\left\{e_{j} \mid j \in \mathbb{Z}\right\}$. It follows that there are no lattice points in the rectangle $Q_{e}$ with the horizontal base, the origin at the lower left vertex, and the diagonal equal to $e_{i}$, shown in Fig. 1. We consider the rotation by $\pi$ which exchanges $J^{u}$ and $J^{u}+e_{i}$.

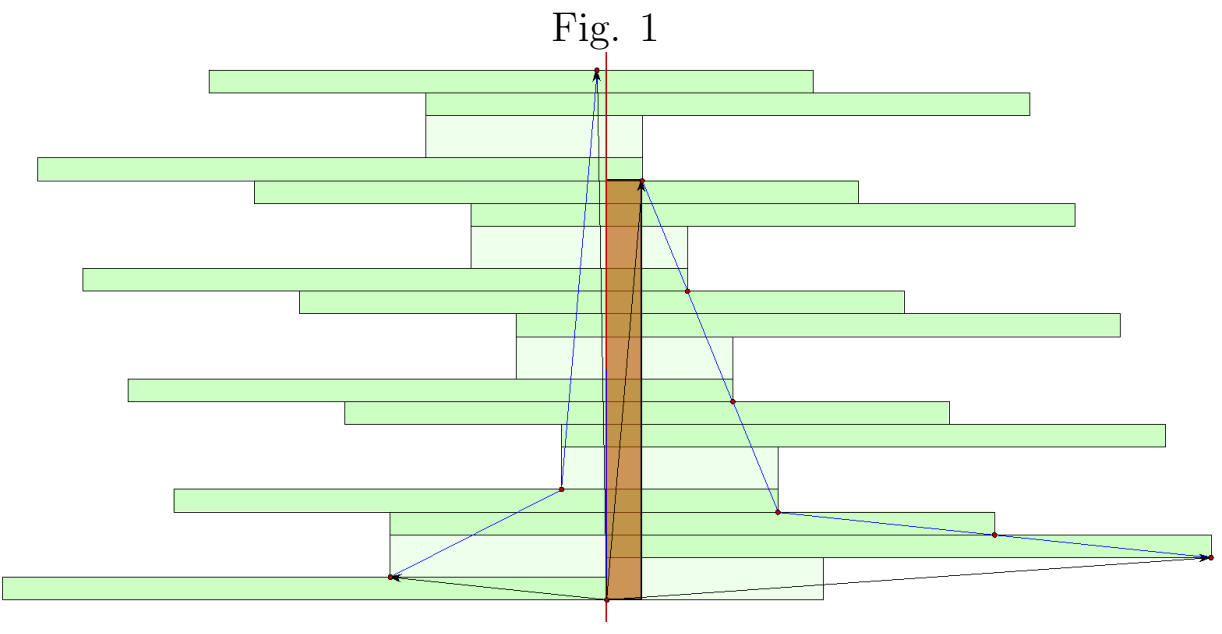

It takes the vertical axis $l_{0}$ into $l_{0}+e_{i}$. As we move the line $l_{0}$ towards $l_{0}+e_{i}$ the part of tiling $\left(c_{0}, c_{1}, c_{2}, \ldots, c_{p-1}\right)$ does not change, since the tilings change only at the crossing of a translated vertical spine and there are none on the way.

Since the principal tiling is palindromic outside of the lock, it follows that $\left(c_{2}, \ldots, c_{p-1}\right)$ must be a palindrome.

The proof for second palindrome requires the introduction of the rectangle $Q_{f}$ with the horizontal base, the origin at the lower right vertex, and the diagonal equal to $f_{i}$. The rest of the argument is the same. Clearly it can also be repeated for $e_{i}+f_{i}$ and the third palindrome. 
To prove the second part let us consider the four rectangles $Q_{e}$, $Q_{f}, Q_{e}+f_{i}$ and $Q_{f}+e_{i}$. They all fit into the rectangle $Q$ with the horizontal base containing the origin, and the other sides containing $e_{i}, f_{i}$ and $e_{i}+f_{i}$, respectively, Fig.2 (note that it has different scalings than Fig.1). Since there are no lattice points in the interior of $Q$, the tilings on the left and right sides of $Q$ differ only by the change in the lock.

Fig. 2

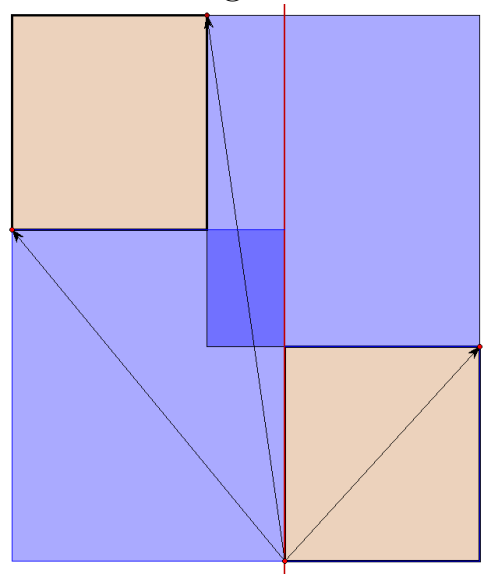

The tilings in $Q_{e}$ and $Q_{e}+f_{i}$, (also in $Q_{f}$ and $Q_{f}+e_{i}$ ) are the same. The segment $l_{0}+e_{i} \cap Q$ is covered by $Q_{e}$ and $Q_{f}+e_{i}$, while the segment $l_{0}+f_{i} \cap Q$ is covered by $Q_{f}$ and $Q_{e}+f_{i}$. It gives us the two representations of the longest palindrome

Let us note that each of the two parts of Theorem 3 allows algorithmic build up of the principal tiling based on the cutting sequence. Indeed let the one sided cutting sequence be equal to $\left(s_{0}, s_{1}, \ldots\right)=$ $(1,1,0,1,1,1,0,0, \ldots$ We get a sequence of vectors starting from $e_{0}=(1,0), f_{0}=(0,1)$ which are the consecutive lattice points in the boundary of Klein's sails in the first and the second quadrants

$$
\left[\begin{array}{l}
1 \\
1
\end{array}\right]\left[\begin{array}{l}
1 \\
2
\end{array}\right]\left[\begin{array}{l}
1 \\
3
\end{array}\right]\left[\begin{array}{l}
2 \\
5
\end{array}\right]\left[\begin{array}{l}
3 \\
8
\end{array}\right]\left[\begin{array}{c}
4 \\
11
\end{array}\right]\left[\begin{array}{c}
5 \\
14
\end{array}\right]\left[\begin{array}{c}
9 \\
25
\end{array}\right]\left[\begin{array}{l}
13 \\
36
\end{array}\right]
$$

They give us all the information necessary to build the palindrome with 12 symbols $a$ and 35 symbols $b$, and we can do it either using the knowledge of the lengths of the palindromes alone, or the second part 
of Theorem 3 .

$b$

$b b$

$b b a b b=b b a b b=b b a b b$

$b b a b b b a b b=b b a b b b a b b=b b a b b b a b b$

$b b a b b b$ a $b b b a b b=b b a b b b a b b b a b b=b b a b b b a b b b a b b$

bbabbbab $b$ babbbabb = bbabbbabbbabb ba $b b=b b a b$ bbabbbabbbabb

bbabbbabbbabbbab babbbabbbabbbabb=

bbabbbabbbabb ba bbabbbabbbabbbabb=

bbabbbabbbabbbabb ab bbabbbabbbabb

bbabbbabbbabbbabbabbbab b babbbabbabbbabbbabbbabb =

bbabbbabbbabb ba bbabbbabbbabbbabbabbbabbbabbbabb =

bbabbbabbbabbbabbabbbabbbabbbabb ab bbabbbabbbabb

In the rational case the fan of bases has the last element $\left(e_{N}, f_{N}\right)$ such that $e_{N}+f_{N}$ is vertical. Accordingly the cutting sequence ends and the tilings are periodic, with the period equal to the sum of components of $e_{N}+f_{N}$. Theorem 3 applies also in this case, and we can obtain the detailed structure of the period as shown in the example.

A segment of the principal tiling containing the vertical spine (the lock) will be called a window. Let us consider one of the palindromes of Theorem 3 of length $k+m-2$ with $k-1$ symbols $a$ and $m-1$ symbols $b$.

Proposition 4. For the principal tiling any window with $k+m$ tiles has $k$ tiles of type $a$ and $m$ tiles of type $b$.

Proof. We have the following structure of the symmetric section of the principal tiling

$$
\left(c_{k+m-1}, \ldots, c_{3}, c_{2},\left[c_{0}, c_{1},\right] c_{2}, c_{3}, \ldots, c_{k+m-1}\right)=\left(w_{1} w_{2}\left[c_{0}, c_{1},\right] w_{1} w_{2}\right)
$$

where $\left(w_{1} w_{2}\right)$ is any split of the palindrome into two words. Hence $\left(w_{2}\left[c_{0}, c_{1},\right] w_{1}\right)$ is a window with $k+m$ tiles and it has the same content as the window $\left(\left[c_{0}, c_{1},\right] w_{1} w_{2}\right)$. 


\section{The nUmber of Berg Partitions}

Recall that a bi-partition is a Berg partition for a toral automorphism $\mathcal{F}$, if $\mathcal{F}\left(J^{s}\right) \subset J^{s}, \mathcal{F}\left(J^{u}\right) \supset J^{u},[\mathrm{~S}-\mathrm{W}]$.

Let us assume that the bi-partition $\left\{R_{1}, R_{2}\right\}$ is a Berg partition for an automorphism $\mathcal{F}$ described in the basis $\left\{e_{0}, f_{0}\right\}$ by $F \in G L(2, \mathbb{Z}), \quad F=$ $\left.\begin{array}{cc}k & l \\ m & n\end{array}\right], k, l, m, n \geq 0$, which preserves the horizontal and vertical

This is the case if $\left(u_{1}, u_{2}\right)$ and $\left(s_{1}, s_{2}\right)$ are, respectively, the row and the column eigenvectors of $F$ with positive entries, [S-W]. If $\lambda>1$ is the unstable eigenvalue of $F$ then $\lambda u_{1}=k u_{1}+m u_{2}, \lambda u_{2}=l u_{1}+n u_{2}$.

The inclusion $\mathcal{F}\left(J^{u}\right) \supset J^{u}$ is obvious since $\mathcal{F}\left(J^{u}\right)=\left[0, \lambda\left(u_{1}+u_{2}\right)\right]$. The other inclusion $\mathcal{F}\left(J^{s}\right) \subset J^{s}$ is also obvious in the case $\operatorname{det} F=1$. However if the stable eigenvalue is negative (in the case $\operatorname{det} F=-1$ ) the origin could be too close to an endpoint for the inclusion to hold. It can be checked by direct estimates that the endpoints of $J^{s}$ are sufficiently far from the origin, but we will establish that by a method based on studying the contents of windows of different sizes, which will be crucial in the following.

Let us introduce the segments of the vertical axis $\alpha_{1}=\left[0, u_{1}\right], \beta_{2}=$ $\left[u_{1}, u_{1}+u_{2}\right], \beta_{1}=\left[0, u_{2}\right], \alpha_{2}=\left[u_{2}, u_{1}+u_{2}\right]$. The vertical spine is split in two ways $J^{u}=\alpha_{1} \cup \beta_{2}=\beta_{1} \cup \alpha_{2}$.

We consider the windows containing the palindromes from Theorem 3 with the number of tiles $p=k+m, r=l+n$ and $p+$ $r=k+m+l+n$, respectively $W_{\alpha}=\left(\left[c_{0}, c_{1}\right], c_{2}, \ldots, c_{p-1}\right), W_{\beta}=$ $\left(\left[c_{0}, c_{1}\right], c_{2}, \ldots, c_{r-1}\right), W_{\gamma}=\left(\left[c_{0}, c_{1}\right], c_{2}, \ldots, c_{p+r-1}\right)$. The window $W_{\alpha}$ has $k$ symbols $a$ and $m$ symbols $b$, so its length is equal to $\lambda u_{1}$. It follows that $\mathcal{F}\left(\alpha_{1}\right)=\left[0, \lambda u_{1}\right]$ is equal to the window $W_{\alpha}$. Similarly $\mathcal{F}\left(\beta_{1}\right)=\left[0, \lambda u_{2}\right]$ is equal to the window $W_{\beta}$. It follows that the endpoints of $\alpha_{1}$ and $\beta_{1}$ are mapped by $\mathcal{F}$ into the horizontal spine $J^{s}$. (Remember that the tilings are obtained by cutting a vertical line by the translates of $J^{s}$.) Since these points are also the endpoints of $J^{s}$ we conclude that $\mathcal{F}\left(J^{s}\right) \subset J^{s}$, and hence the bi-partition is a Berg partition.

There are translates of $\left\{R_{1}, R_{2}\right\}$ which are also Berg partitions. We will count the number of such translates, up to the following equivalence. We say that two Berg partitions $\left\{Q_{1}, Q_{2}\right\}$ and $\left\{S_{1}, S_{2}\right\}$ for the toral automorphism $\mathcal{F}$ are equivalent if there is a toral affine map $\mathcal{U}$ such that

$$
\mathcal{F} \circ \mathcal{U}=U \circ \mathcal{F}, \quad \mathcal{U}\left(Q_{i}\right)=S_{i}, i=1,2 .
$$

Note that any homeomorphism commuting with a hyperbolic toral automorphism must be an affine map, $\mathrm{A}-\mathrm{P}$.

Theorem 5. The number of nonequivalent Berg partitions for $\mathcal{F}$ which are translates of the bi-partition $\left\{R_{1}, R_{2}\right\}$ is equal to $\left[\frac{k+m+l+n}{2}\right]$. 
Proof. Instead of translating the bi-partition we fix the rectangles $\left\{R_{1}\right.$, $\left.R_{2}\right\}$ in the affine plane and translate the origin inside $J^{u}$. We get immediately $\mathcal{F}\left(J^{u}\right) \supset J^{u}$, and if under such a translation the bi-partition is still a Berg partition then $\mathcal{F}\left(J^{u}\right)$ is the window $\widehat{W}_{\gamma}$ of the principal tiling with $p+r$ tiles, which by Proposition 4 has the same contents as the window $W_{\gamma}$.

Conversely let us consider a window $W$ with $p+r$ tiles, e.g., $W_{\gamma}$ is such a window. There are $p+r-1$ such windows, and by Proposition 4 they have the same contents, i.e., they contain $k+l$ tiles of type $a$ and $m+n$ tiles of type $b$. It follows that their lengths are the same and equal to $\lambda\left(u_{1}+u_{2}\right)$. For a given window $W$ we place the origin in $J^{u}$ in such a way that $\mathcal{F}\left(J^{u}\right)=W$. We claim that in this way we will obtain a Berg partition, that is we need to prove that $\mathcal{F}\left(J^{s}\right) \subset J^{s}$. By the construction the endpoints of $J^{u}$ belong to $J^{s}$ and are mapped to the endpoints of the window $W$. Since a tiling is a partition of a vertical line by the translates of $J^{s}$ we conclude that the endpoints of $J^{u}$ are mapped into $J^{s}$. We need to explore the image of the endpoints of $J^{s}$. These two points split the lock $J^{u}$ in two ways, $\alpha_{1} \cup \beta_{2}=\beta_{1} \cup \alpha_{2}$, or in symbolic representation $[a, b]$ and $[b, a]$.

Accordingly let us examine the split of the window $W$ into two segments $W=w_{1} \cup w_{2}$, containing $p$ and $r$ tiles respectively. One of the segments will be a window itself, with the exception in the case when the common endpoint of $w_{1}$ and $w_{2}$ splits the lock.

If say $w_{1}$ is a window then by Proposition 4 it has the same contents as $W_{\alpha}$, i.e., $k$ symbols $a$ and $m$ symbols $b$. It follows that the segment $w_{2}$ (although it is not a window itself) has the same contents as $W_{\beta}$. If the common point of $w_{1}$ and $w_{2}$ falls into the lock $J^{u}$, then we can exploit the ambiguity in the splitting of the lock, and choose one so that $w_{1}$ and $w_{2}$ have the same contents as $W_{\alpha}$ and $W_{\beta}$. We conclude that the length of $w_{i}$ is $\lambda u_{i}, i=1,2$, and $\mathcal{F}\left(\alpha_{1}\right)=w_{1}, \mathcal{F}\left(\beta_{2}\right)=w_{2}$. Hence the endpoint $\left(0, u_{1}\right)$ of $J^{s}$ (in $\mathbb{T}^{2}$ ) is mapped into the endpoint of $w_{1}$, which belongs to $J^{s}$.

We can repeat this argument splitting the window $W$ into two segments $w_{1}$ and $w_{2}$ with reversed contents, $r$ tiles and $p$ tiles, respectively. We will conclude then that also the other endpoint $\left(0, u_{2}\right)$ of $J^{s}$ is mapped into $J^{s}$. It follows that $\mathcal{F}\left(J^{s}\right) \subset J^{s}$ and our bi-partition is a Berg partition.

For different choices of the window $W$ we obtain different locations of the origin in $J^{u}$. Two of thus obtained Berg partitions are equivalent if and only if the respective locations of the origin in $J^{u}$ are symmetric. Indeed, any toral affine map $\mathcal{U}$ preserving the bi-partition $\left\{R_{1}, R_{2}\right\}$ must map $J^{u}$ onto itself, and the fixed point contained there into the other fixed point. It happens only for $\mathcal{U}$ equal to the rotation by $\pi$ around the center of $J^{u}$, and the two fixed points located symmetrically inside $J^{u}$. 
We conclude that the number of nonequivalent Berg partition is equal to $\frac{p+r-1}{2}$ if $p+r$ is odd, and $\frac{p+r}{2}$ if $p+r$ is even.

We can visualize the different Berg partitions by considering the refinement of the 2-periodic tiling of $\mathbb{R}^{2}$ and its preimage under $\mathcal{F}$. In other words we consider the partition of the torus $\mathbb{T}^{2}$ by $J^{u}$ and $\mathcal{F}^{-1}\left(J^{s}\right)$. This partition is mapped onto the partition of the torus by $J^{s}$ and $\mathcal{F}\left(J^{u}\right)$. These partitions are shown in Fig. 3 for the matrix $F=\left[\begin{array}{ll}5 & 2 \\ 7 & 3\end{array}\right]$, and the origin at the lower endpoint of $J^{u}$. Let us note that they are generating Markov partitions for the toral automorphism. The number of wide and narrow horizontal rectangles which split $R_{1}$

Fig. 3

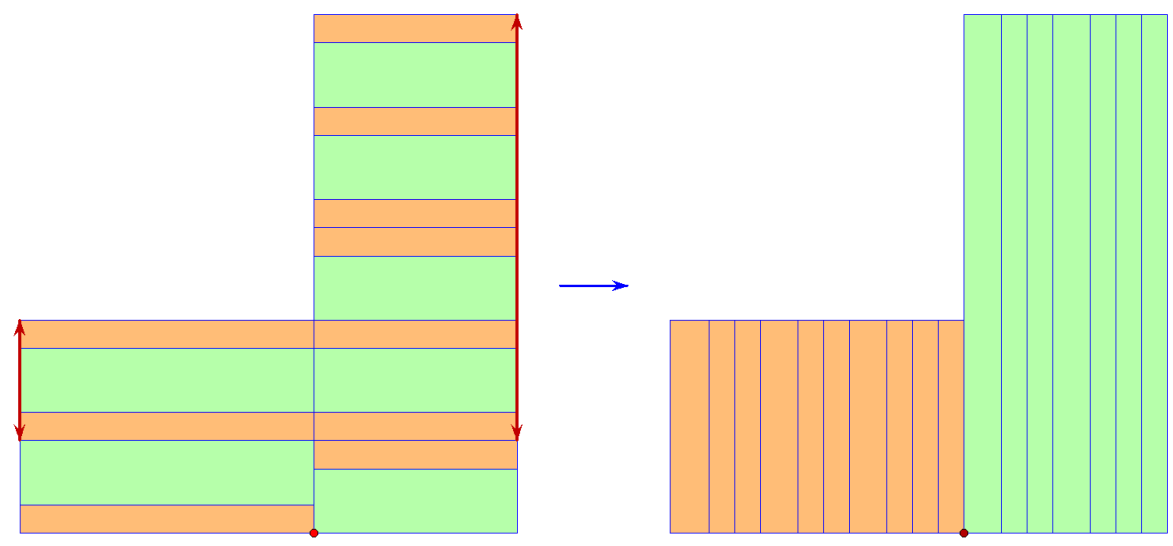

and $R_{2}$ are equal to the respective entries of the matrix $F$, and is the same for all Berg partitions. However their order is different for different Berg partitions. The order is the same as the order of tiles in the two segments $w_{1} \cup w_{2}=\mathcal{F}\left(J^{u}\right)$ from the proof of Theorem 5, with $p=k+m$ and $r=l+n$ tiles respectively. The ambiguity in the lock is resolved by the following rule. For $\operatorname{det} F=1$ the lock is chosen as $[a, b]$, and for $\operatorname{det} F=-1$ it is chosen as $[b, a]$.

We can see that the connectivity matrix for a Berg partition is equal to $F^{T}$. Theorem 1, proven in [S-W], is somewhat more general than Theorem 5. It claims that for any toral automorphism the number of nonequivalent Berg partitions with a given connectivity matrix is equal to half the sum of its entries. It can be derived from Theorem 5 as follows.

It was proven in $[\mathrm{S}-\mathrm{W}]$ that a hyperbolic toral automorphism is given by a matrix $F \in G L(n, \mathbb{Z})$ with nonnegative entries, or $-F$, if and only if the chosen basis of the lattice $L$ is from the fan of bases associated with the stable and unstable lines. If the automorphism $\mathcal{F}$ is given by $F$ with non-negative entries then Theorem 5 applies.

It remains to consider the case of the toral automorphism which is given by $-F$. Let $\mathcal{S}$ be the rotation by $\pi$ around the centers of the 
bi-partition $\left\{R_{1}, R_{2}\right\}$, associated with the chosen basis $\left(e_{0}, f_{0}\right)$ of the lattice $L$. As in the proof of Theorem 5, we fix the bi-partition and translate the origin to define $\mathcal{F}$ with the representation $F$. However $\mathcal{S}$ is tied to the bi-partition, and not to the shifting origin. For any choice of the origin in $J^{u}$, consider the mapping $\mathcal{F} \circ \mathcal{S}$. It has a fixed point in $J^{u}$, different from the origin, unless the origin falls into the center of $J^{u}$. If we place the origin in this fixed point, the mapping $\mathcal{F} \circ \mathcal{S}$ becomes a toral automorphism given by the matrix $-F$ in the basis $\left(e_{0}, f_{0}\right)$, and the bi-partition is its Berg partition. Indeed $S\left(J^{u}\right)=J^{u}, S\left(J^{s}\right)=J^{s}$, and hence $\mathcal{F} \circ \mathcal{S}\left(J^{u}\right) \supset J^{u}$ and $\mathcal{F} \circ \mathcal{S}\left(J^{s}\right) \subset J^{s}$. Moreover the connectivity matrices for the Berg partitions are the same.

In this way we put the Berg partitions of $\mathcal{F}$ and $\mathcal{F} \circ \mathcal{S}$ into 1-1 correspondence. We can conclude that the number of Berg partitions with the connectivity matrix $F^{T}$, for the toral automorphisms $\mathcal{F}$ and $\mathcal{F} \circ \mathcal{S}$ is the same.

\section{Substitution tilings}

We have seen that a Berg partition for a toral automorphism $\mathcal{F}$ comes with the distinctive pattern of the generating Markov partition, with the boundaries equal to $J^{u} \cup \mathcal{F}^{-1}\left(J^{s}\right)$, Fig 2 . These patterns are associated with substitutions preserving the tilings. We will describe this in detail.

The toral automorphism $\mathcal{F}$ acts on $\mathbb{T}^{2}$, which for any fixed bi-partition can be interpreted as the space of tilings. If the underlying bi-partition is a Berg partition then on the symbolic level the action of $\mathcal{F}$ is by substitutions. More precisely let us consider the palindromes from Theorem 3 with lengths $p-2=k+m-2$ and $r-2=l+n-2$, respectively $\alpha^{\prime}=\left(c_{2}, \ldots, c_{p-1}\right)$ and $\beta^{\prime}=\left(c_{2}, \ldots, c_{r-1}\right)$.

We consider the substitutions

$$
\begin{array}{rlrl}
a & \mapsto \alpha=[a, b] \alpha^{\prime}, & & a \mapsto \alpha=[b, a] \alpha^{\prime}, \\
b & \mapsto \beta=[b, a] \beta^{\prime}, & b \mapsto \beta=[a, b] \beta^{\prime},
\end{array}
$$

the left one if $\operatorname{det} F=1$, and the right one if $\operatorname{det} F=-1$. We will call such a substitution the standard substitution.

The principal tiling is invariant under the standard substitution. More generally for a tiling on the vertical line passing through a point $h \in J^{s}$ the standard substitution produces the tiling on the line through the point $\mathcal{F}(h) \in J^{s}$. Further for any vertical line containing a fixed point of $\mathcal{F}$ the corresponding tiling will be invariant under the standard substitution, up to the shift. It means that after the substitution the new tiling is the same as old, when viewed from the appropriate place, determined by the location of the fixed point.

Shifting the origin in $J^{u}$, as in the proof of Theorem 5, we obtain another Berg partition, and also another substitution. It is defined by the splitting of the window $W=\mathcal{F}\left(J^{u}\right)=w_{1} \cup w_{2}$, where $w_{1}$ has 
$p=k+m$ tiles and $w_{2}$ has $r=l+n$ tiles. The substitution is $a \mapsto w_{1}, b \mapsto w_{2}$, with the provision that for $\operatorname{det} F=1$, the lock is chosen as $[a, b]$, and for $\operatorname{det} F=-1$ it is chosen as $[b, a]$.

There are at most $p+r-1$ of these substitutions, according to the choice of the window $W$. All of them must be actually different. Indeed we obtain them from the standard substitution by cyclic permutations of the words $\alpha$ and $\beta$. Should two different cyclic permutations of $\alpha$ produce the same word, then the number of symbols $a$ (equal to $k$ ) and the number of symbols $b$ (equal to $m$ ) would have to have a common factor, which is not the case. Since also $p$ and $r$ are relatively prime there is no common multiple of $p$ and $r$ smaller than $p+r$. We conclude that all of the $p+r-1$ cyclic permutations of $\alpha$ and $\beta$ deliver different pairs of words, and hence different substitutions.

The counting of Berg partitions is different from the counting of substitutions since the toral affine map $\mathcal{S}$ defined above conjugates two Berg partitions with reversed substitutions.

Séébold [Seb] proved that there are exactly $k+l+m+n-1$ combinatorial substitutions which preserve Sturmian words associated with a given matrix $F \in G L(2, \mathbb{Z})$ with nonnegative entries.

Combining this result with Theorem 5 we conclude that all these combinatorial substitutions can be realized geometrically as Berg partitions with the connectivity matrix $F^{T}$. It follows that any question about the properties of Sturmian sequences with substitution symmetry becomes a question about Berg partitions for the respective toral automorphism.

\section{REFERENCES}

[A-P] R. L. Adler, R. Palais, Homeomorphic conjugacy of automorphisms on the torus. Proc. Amer. Math. Soc. 16 (1965), 1222-1225.

[H-M1] M. Morse, G.A. Hedlund, Symbolic Dynamics. Amer. J. Math. 60 (1938), no. 4, 815-866.

[H-M2] M. Morse, G.A. Hedlund, Symbolic dynamics II. Sturmian trajectories. Amer. J. Math. 62 (1940), 1-42.

[L-M] A. de Luca, F. Mignosi, Some combinatorial properties of sturmian words. Theoretical Computer Science 136 (1994), 361-385.

[P] A. Pedersen, Solution of Problem E 3156. The American Mathematical Monthly 95 (1988), 954-955.

[PF] N. Pytheas Fogg, Substitutions in Dynamics, Arithmetics and Combinatorics. Lecture Notes in Mathematics 1794. Springer-Verlag Berlin 2002.

[R] P. M. Robinson, Problem E 3156. The American Mathematical Monthly 93 (1986), 482.

[Seb] P. Séébold, On the conjugation of standard morphisms. Mathematical foundations of computer science (Cracow, 1996). Theoret. Comput. Sci. 195 (1998), no. $1,91-109$.

[Ser] C. Series, The geometry of Markoff numbers. Math. Intelligencer 7 (1985), no. 3, 20-29.

[S-W] A. Siemaszko, M. P. Wojtkowski, Counting Berg partitions. Nonlinearity 24 (2011) 2383-2403. 
Department of Mathematics and Computer Science University OF WARMia AND MaZURY IN OLSZTYN

SEONECZNA 54

10-710 OLSZTYN, POLAND

E-mail address: artur@uwm.edu.pl, wojtkowski@matman.uwm.edu.pl 